\title{
Exploring the Determinants of Suicidal Behavior: Conventional and Emergent Risk (DISCOVER): a feasibility study
}

Zainab Samaan 1,2,3,4,5,6*, Monica Bawor ${ }^{3,4}$, Brittany B. Dennis ${ }^{2,3}$, Wala El-Sheikh ${ }^{5}$, Jane DeJesus ${ }^{5}$, Sumathy Rangarajan ${ }^{5}$, Judith Vair ${ }^{6}$, Heather Sholer ${ }^{6}$, Nicole Hutchinson ${ }^{6}$, Elizabeth Iordan ${ }^{6}$, Pam Mackie $^{5}$, Shofiqul Islam ${ }^{5}$, Mahshid Deghan ${ }^{5}$, Jennifer Brasch ${ }^{1,6}$ and Lehana Thabane $2,5,6,7,8$

\begin{abstract}
Background: Suicidal behavior is a growing public health concern resulting in morbidity and premature death. Although certain factors such as age, sex, and psychiatric disorders have been consistently reported to be associated with suicidal behavior, other factors including biological markers, diet, and physical activity may also influence suicidal behavior. The purpose of this pilot study was to evaluate the feasibility of conducting a full-scale study to identify the conventional and novel risk factors of suicidal behavior in individuals who made a recent suicide attempt.

Methods: This pilot study was a case-control study of participants with recent (within 1 month of admission) suicide attempts admitted to hospital and compared to two control groups: 1) psychiatric inpatient participants without a history of suicide attempts and 2) community-based controls. We collected information on demographic variables, circumstances of suicide attempts (for cases), medical and psychiatric diagnoses, behavioral patterns, physical measurements, and social factors. Blood and urine samples were also collected for biological markers. Feasibility outcomes are as follows: 1) $50 \%$ of all eligible cases will consent to participate, 2) 50 cases and 100 controls per year can be recruited, and 3) at least $80 \%$ of the participants will provide blood samples for DNA and biological markers.
\end{abstract}

Results: We recruited 179 participants in total; 51 cases, 57 psychiatric controls without suicide attempt, and 71 non-psychiatric controls in Hamilton, Ontario. Recruitment rate was 70 \% (213/304), and we obtained urine and blood specimens from $90 \%$ (191/213) of participants. Questionnaire completion rates were high, and data quality was very good with few data-related queries to resolve. We learned that cases tended to be hospitalized for long periods of time and the suicide attempt occurred more than a month ago in many of the cases; therefore, we expanded our inclusion criterion related to timing of suicide attempt to 3 months instead of 1 month.

Conclusions: The study procedures needed certain modifications including extending the time between suicide attempt and date of recruitment, and more detailed questionnaires related to diet were necessary while other questionnaires such as social support needed to be shortened. Overall, this study showed that it is feasible to conduct a larger-scale study.

Keywords: Pilot study, Suicide, Suicidal behavior, Risk factors, Biomarkers, Social determinants

\footnotetext{
* Correspondence: samaanz@mcmaster.ca

'Department of Psychiatry and Behavioural Neurosciences, McMaster

University, Hamilton, ON, Canada

²Department of Clinical Epidemiology and Biostatistics, McMaster University,

Hamilton, ON, Canada

Full list of author information is available at the end of the article
} 


\section{Background}

Suicide is a leading cause of premature death worldwide, with over one million people dying by suicide each year [1]. Data from the World Health Organization shows that suicide accounts for $1.4 \%$ of total deaths globally [2]. In Canada, the rate of completed suicide is estimated at 11.3 per 100,000 individuals with men three times more likely to die by suicide than women [2].

Suicidal behavior (SB) is a complex set of ideas, plans, and acts intended to end one's life and is 10-20 times more common than completed suicide [3]. SB is reported to be among the most important risk factors for suicide [4-6]; however, it tends to be underreported due to several factors including a lack of recognition by services, denial of attempts, avoidance of legal inquiries, access to health services, stigma attached to suicidal behavior, financial consequences such as insurance claims, and lack of a national registry of SB. Over $90 \%$ of individuals with SB have an identifiable psychiatric disorder [7]; however, not all patients with psychiatric disorders attempt suicide, suggesting a "diathesis" or predisposition to SB in susceptible individuals independent of underlying psychopathology [3].

Several demographic, biological, behavioral, and social factors influence $\mathrm{SB}$, and it is likely that multiple other known and unknown factors contribute to SB. The interaction between environmental and biological factors is likely to provide a plausible understanding of the risk factors of suicide; however, this will require a refined phenotypic characterization of suicidal behavior and associated behaviors. The existing studies of SB provide valuable information on the conventional risk factors of suicide (such as age and sex); however, other risk factors are less investigated. For instance, many studies have sought to evaluate the links between diet and suicidal behavior. Increased suicide rates in people with low cholesterol or after lowering cholesterol through diet have been reported [8-13]. Studies of eicosapentaenoic acid (EPA), a polyunsaturated fatty acid found in fish oil, report a significant association between low EPA and SB [14]. Japanese studies reported lower risk of suicide in individuals consuming fish regularly [15]. Few studies have sought to determine the impact of exercise on suicidal behavior by demonstrating a direct link between exercise and suicide risk [16]. Thus, other dietary factors as well as exercise behaviors may also have a significant contribution to $\mathrm{SB}$ and are worth exploring further.

While several studies report risk factors associated with completed suicide [17], the risk factors associated with suicide attempts are likely to be different. In a study of genetic variants associated with suicide, suicide attempts were associated with a different genetic profile than completed suicide [18]. This suggests that SB is not a spectrum of severity phenomenon with suicide ideas on the milder end and completed suicide on the severe end, but likely to be better characterized as discrete categories with different risk factors for each category. Understanding both the inherent and modifiable risk factors of SB will assist in the prevention of $\mathrm{SB}$ and therefore completed suicide as well.

The main purpose of the Determinants of Suicide: Conventional and Emergent Risk (DISCOVER) study is to understand what risk factors are associated with suicide attempts that required hospitalization. We aimed to provide a comprehensive evaluation and phenotypic characterization of individuals with suicide attempts, and we propose a theoretical model of risk factors (Fig. 1) and how the interaction of these factors can lead to SB. We aim to systematically investigate conventional risk factors (including demographic variables, presence of chronic illness, socioeconomic factors, family history of suicide, and previous hospitalization) [19-21] as well as promising emergent risk factors (biological markers including peripheral levels of neurotransmitters [22, 23], chemokines [24], and genetic variants [18]; diet [25, 26]; vitamin D deficiency [27]; smoking [28]; and physical activity [29]) by designing a case-control study of individuals who made a recent (within 1 month of admission) suicide attempt and comparing them to two types of controls, psychiatric patients admitted to hospital without history of suicide attempts and non-psychiatric community-based controls.

The principal objective of this pilot study is to assess the overall feasibility of the main DISCOVER study by evaluating the following outcomes: 1) recruitment rate, 2) adherence to study procedures including completion of questionnaires and fasting for blood and urine samples, 3) feasibility of genetic and laboratory data collection, 4) preliminary evidence on emergent risk factors, and 5) assessment of overall study procedures including eligibility criteria.

\section{Methods}

Eligible participants were recruited for the pilot study between March 2011 and December 2012 in Hamilton, Ontario, a mid-size Canadian city. The main aim of this pilot and feasibility study was to enhance the likelihood of success of the full-scale study. The objectives of this study are grouped into three main domains after Thabane and colleagues [30]: 1) to assess the feasibility of the study processes including the recruitment rate, refusal rate, adherence to overnight fasting procedure, eligibility criteria, understanding of study questionnaires; and 2) to establish resources needed as well as assess any management-related issues such as access to a private interview room when needed, and data management challenges such as missing variables and data variability. In addition we wanted to explore preliminary evidence for biological markers of suicide attempts. 


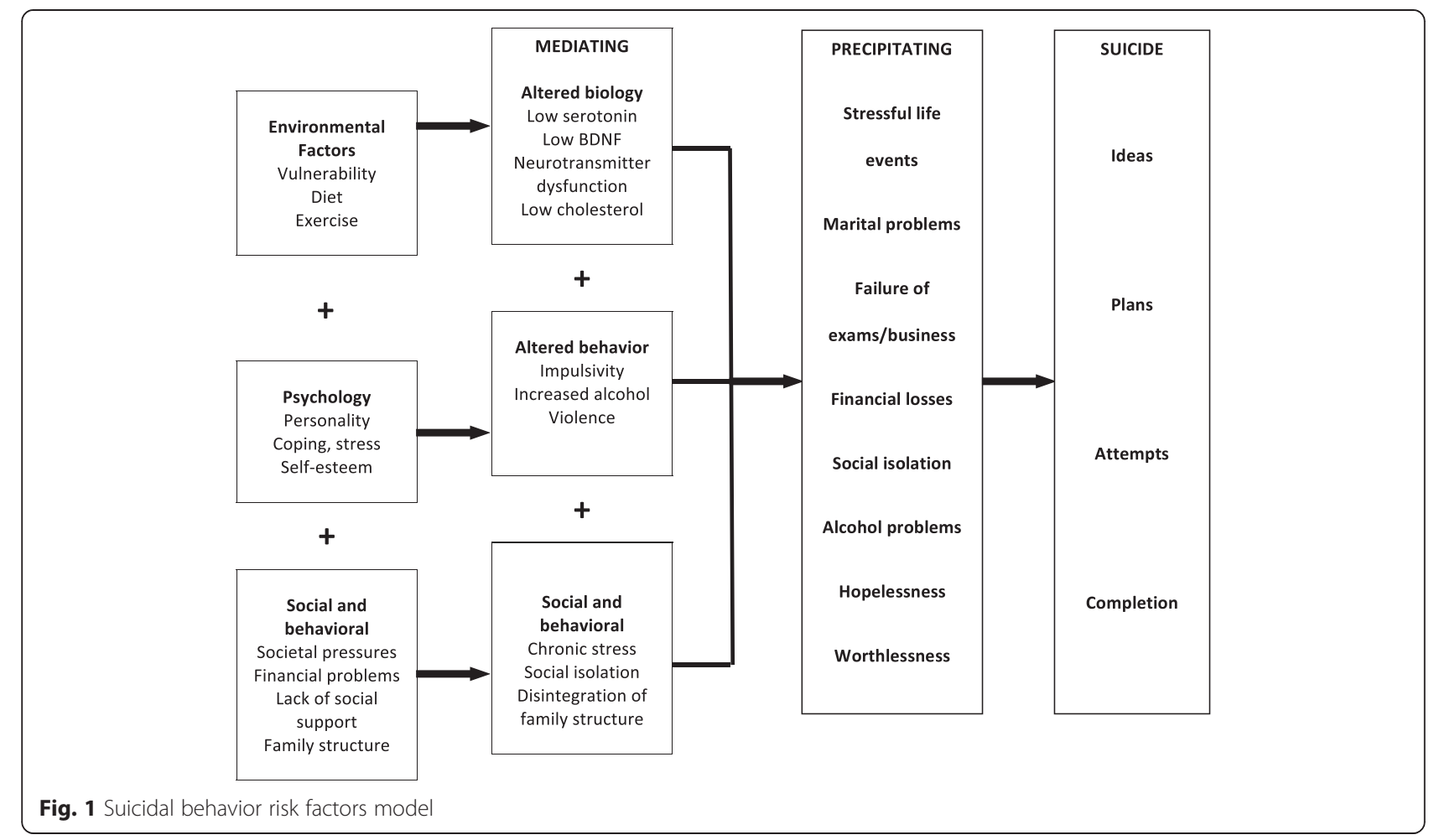

We performed a case-control study of hospitalized individuals who attempted suicide within the past month and compared them to two control groups; one group consisting of hospitalized individuals with psychiatric disorders who did not have any history of suicide attempts, and another group consisting of individuals attending the general hospital for minor medical procedures, admitted to the hospital for medical/surgical causes, attending outpatient clinics, or community-based and not seeking health care.

\section{Criteria for assessing feasibility}

We used the following parameters to assess the success of the feasibility study: 1) $50 \%$ of all eligible cases consent to participate, 2) 50 cases and 100 controls per year can be recruited, and 3) at least $80 \%$ of the participants will provide blood samples for DNA and biological markers.

\section{Setting and participants}

The inclusion criteria for the pilot study were individuals aged 18 years or older who were able to provide written informed consent, communicate in English, and follow study procedures. The cases were individuals who made a recent serious (within past month) suicide attempt, which we defined as a suicide attempt that required med$\mathrm{ical} /$ psychiatric intervention and admission to psychiatric or medical wards, and were inpatients at the time of study enrolment. To be defined as a case, patients were required to indicate they intended to die as a result of their self- harm. The cases were age (within 5 years) and sex matched to two controls. Control group 1 (control 1) participants were eligible for the study if they had psychiatric disorders without current or past history of suicide attempts and were admitted to the psychiatric hospital within the same time frame as the cases. Control group 2 (control 2) participants were recruited from the general population in the community or general hospitals and were included in the study if they did not have any history of suicide attempts.

Exclusion criteria include inability to provide informed consent or to follow study procedures. Given the heterogeneous nature of suicidal behavior, we defined a case as a person who made a suicide attempt that required hospital admission or who was considered based on clinical assessment at the time of presentation to have made a suicide attempt requiring hospital treatment. Potential study participants were approached by trained research personnel and detailed information about the study was provided. Individuals who agreed to participate in the study were asked to sign written informed consent. Each participant was interviewed using a structured interview with validated rating scales and questionnaires. All individuals underwent a psychiatric diagnostic interview and physical measurements, and blood and urine samples were obtained after an overnight fast. The pilot study was approved by the Hamilton Health Sciences (\#10-661) and St. Joseph's Healthcare (\#11-3479) Research Ethics Boards. 
Researchers for the inpatient recruitment approached the clinical staff and asked about suitable participants based on the study inclusion criteria. Clinical staff identified suitable patients after obtaining verbal consent from patients to be approached by research staff. Research staff would then obtain patient consent for participants. Upon obtaining consent, patients were asked questions pertaining to suicide attempt history, and medical records were used to confirm patients' response.

For community recruitment, the researchers distributed advertisements in hospitals and community requesting volunteers to participate in the study. Upon obtaining consent, the researcher would take a history of suicide attempt and mental health from community participants. All participants were subject to screening and diagnostic interviewing for psychiatric disorders using the M.I.N.I. diagnostic assessment. Medical history was also obtained from all patients, including history of attending clinical services and current medications. The medical charts of included participants attending medical or surgical services were also checked for confirmation of diagnoses and history.

\section{Data collection and instruments}

The DISCOVER study questionnaires were designed to acquire information on participants' risk factors for suicide in a face-to-face interview format. We obtained data on socio-demographic variables (age, sex, ethnicity, religion, marital status, education, employment and current social support), medical history and current medications, diet, impulsivity, psychopathology, suicidal behavior (number of past attempts, details of methods used, age at first attempt, and family history of suicide), and physical measurements (blood pressure, pulse rate, height, weight, hip and waist circumference). The study questionnaires were compiled utilizing previously validated diagnostic and assessment tools including: Beck Suicide Intent Scale [31], M.I.N.I. International Neuropsychiatric Interview (Version 6.0) [32], Barratt Impulsivity Scale [33], Social Support Questionnaire [34], Borderline Symptom List 23 [35], Physical Activity Questionnaire [36, 37], and a short Food Frequency Questionnaire (FFQ) [38]. The FFQ consists of a food list, pre-determined portion size, and frequency of intake.

The study questionnaires were administered in hospital or community by trained research staff and subsequently entered into a confidential database at the project management centre of the Population Health Research Institute (PHRI). Fasting blood and urine samples were also obtained. There was no consultation with patients during the design of study. However, nurses provided feedback during study meetings on the processes, case report forms, as well as time it took to recruit and interview patients. Specific attention was paid to any feedback on patients' comments during the interview, particularly whether patients found them long, tiring, and whether any questions were difficult to understand. Revisions were made to our questionnaires based on this feedback. For example, we changed the wording on one of our diet questionnaires from "rather much" to "often".

We adhered to the standard consenting procedures and ethical practices outlined in the Declaration of Helsinki. During the consenting process, patients were informed of the study purpose, procedures, potential benefits, possible harms, as well as their ability to withdraw at any time. Participants were also required to consent to the collection and storage of biological material (blood samples). Regardless of reason, interviews were stopped at any times patients wished to withdraw.

Patients approached to participate could be voluntary (informal) or admitted under a form using the Canadian mental health act (formal). The Mental Health Act (MHA) in our jurisdiction does not automatically consider patients that were admitted informally to provide consent. In our system, patients can be admitted formally and still be considered competent to consent to treatment. Therefore, the patients included in this study contained a mixture of both informal and formal admissions; however, all patients are deemed competent to consent. Patients who are considered incompetent to consent to treatment are assessed using the MHA and would have a substitute decision maker to make decisions on their behalf. We did not approach patients who were deemed incompetent to consent. People who are assessed using MHA considered incompetent are usually patients with confusion, cognitive impairment, or severe psychotic symptoms impairing judgment, and therefore considered unable to make informed decisions.

\section{Statistical analysis}

For the pilot study, we provide descriptive statistics expressed as mean and standard deviation (SD) for continuous variables and number (percent) for categorical variables. We used the Analysis of Variance (ANOVA) technique to compare means across different subgroups and $\chi^{2}$ test to compare proportions for categorical variables. Results from the ANOVA may suggest a difference exists between groups; however, we are unable to determine which groups are contributing to the difference. To circumvent this problem, we also performed a pairwise comparison and we reported the mean differences between cases versus control group 1 and cases versus control group 2. We have included these additional analyses in a supplementary appendix.

\section{Laboratory methods}

Blood samples were collected, and after $30 \mathrm{~min}$ of clotting time, tubes were spun at $1500 \times g(3000 \mathrm{rpm})$ for 15 
min until blood was well separated (with the exception of the PAXgene tube). Plasma and serum samples were then aliquoted and stored in cryovials at the data collection site within $2 \mathrm{~h}$ of collection, then frozen in liquid nitrogen $\left(-196{ }^{\circ} \mathrm{C}\right)$ at the Clinical Research and Clinical Trials Laboratory, Hamilton. Urine samples were also aliquoted and stored in liquid nitrogen $\left(-196{ }^{\circ} \mathrm{C}\right)$ at the Clinical Research and Clinical Trials Laboratory for future analyses.

\section{Results}

\section{Feasibility of the study processes}

We recruited participants from multiple sources (psychiatric hospital, general hospital, clinics and community) in Hamilton, Ontario, Canada, over a 20-month period from March 2011 to December 2012. In total, 213 participants were recruited (see Fig. 2 for participant flow diagram).

During the pilot study, we approached 304 individuals and enrolled 213 (70\%). Of the individuals enrolled in the study, 51 subjects had made a recent serious suicide attempt, 57 participants were in control group 1, 71 were in control group 2, and 34 individuals were individuals with past history of suicide attempts (this group was not included in the pilot study data analyses). The characteristics of the study participants are provided in Table 1, where we also present the differences between three groups of participants (cases, control 1, and control 2) using ANOVA. The mean differences between cases versus control group 1 and cases versus control group 2 can be found in Additional file 1: Table S1 and Additional file 1: Table S2 of the supplementary appendix.

\section{Establishing resources needed and assessment of study management}

We were able to recruit 213 participants during a 20 month period, equivalent to a $70 \%$ recruitment rate (304 potential participants approached and 213 participants recruited), demonstrating one of the feasibility outcomes for the full-scale study. Most participants (191 out of 213 participants (90\%)) provided early morning (between 7:009:00 am) fasting blood samples according to the study protocol, which ensures that biological testing will be feasible. Completion rates for the questionnaires were very high (>90\%), which suggests that our data collection and interview process are feasible to obtain and complete all the necessary information for the study (Table 2).

There were few reasons patients did not provide blood samples: participants did not return to the appointment to provide samples, they did not have the time to return, they were discharged, or we were unable to obtain their blood.

Research staff reported some difficulties when administering the full questionnaire, particularly the length of the interview process. Both interviewers and participants reported being unable to stay focused for the duration of the interview, or being interrupted by scheduled tasks for hospitalized participants.

\section{Preliminary results}

The findings of this pilot study demonstrate that demographic factors including marital status, education level, employment status, and current smoking differed significantly between cases and controls (Table 1). The percentage of employed participants was lower among cases compared to both controls (unadjusted $\chi^{2}=13.3$, $\mathrm{df}=2, p=0.001$ ) and the percentage of current smokers was significantly greater among cases compared to control groups (unadjusted $\chi^{2}=22.6, \mathrm{df}=4, p<0.001$ ).

Preliminary results of the physical activity and leisure patterns for the past 12 months from the pilot data are shown in Fig. 3. Interesting trends are seen with cases being mostly sedentary compared to controls (unadjusted $\chi^{2}=18.1, \mathrm{df}=6, p=0.006$ ).

We looked at dietary patterns for the past 12 months and our preliminary results showed that cases consumed less whole grains, vegetables, and fruits per day and less fish and nuts per week than controls (Fig. 4). Significant group differences between cases and controls were found for nut consumption per week $(F(2,158)=6.07, p=0.003)$ and a trending pattern was found for raw vegetable consumption $(F(2,158)=3.00, p=0.052)$. The error lines in this figure represent variance around the mean estimates for number of food servings. These lines extend to the outer fences of interquartile range, which is represented by the size of the box. Any dots in this image represent outlier observations.

\section{Discussion}

The existing literature on risk factors of SB has identified multiple conventional risk factors for suicide; however, these studies are limited by modest sample size, inadequate characterization of the sample, and the inclusion of individuals with life-time history of suicidal attempts (retrospective data). Several studies lacked a control group and studies report conflicting findings. In addition, several studies adopted a nested case control design using convenience samples and investigated suicide attempts in a specific population based on psychopathology or other denominators. We aim to address these limitations with the design of the DISCOVER study; however, we opted to complete a pilot and feasibility study to enhance the likelihood of success of the main study.

This DISCOVER pilot study demonstrated feasibility to conduct the main study across many aspects including recruitment, participants' willingness to provide blood and urine samples, questionnaire completeness, and encouraging data trends including confirmation of previously known risk factors. We would like to emphasize that the 


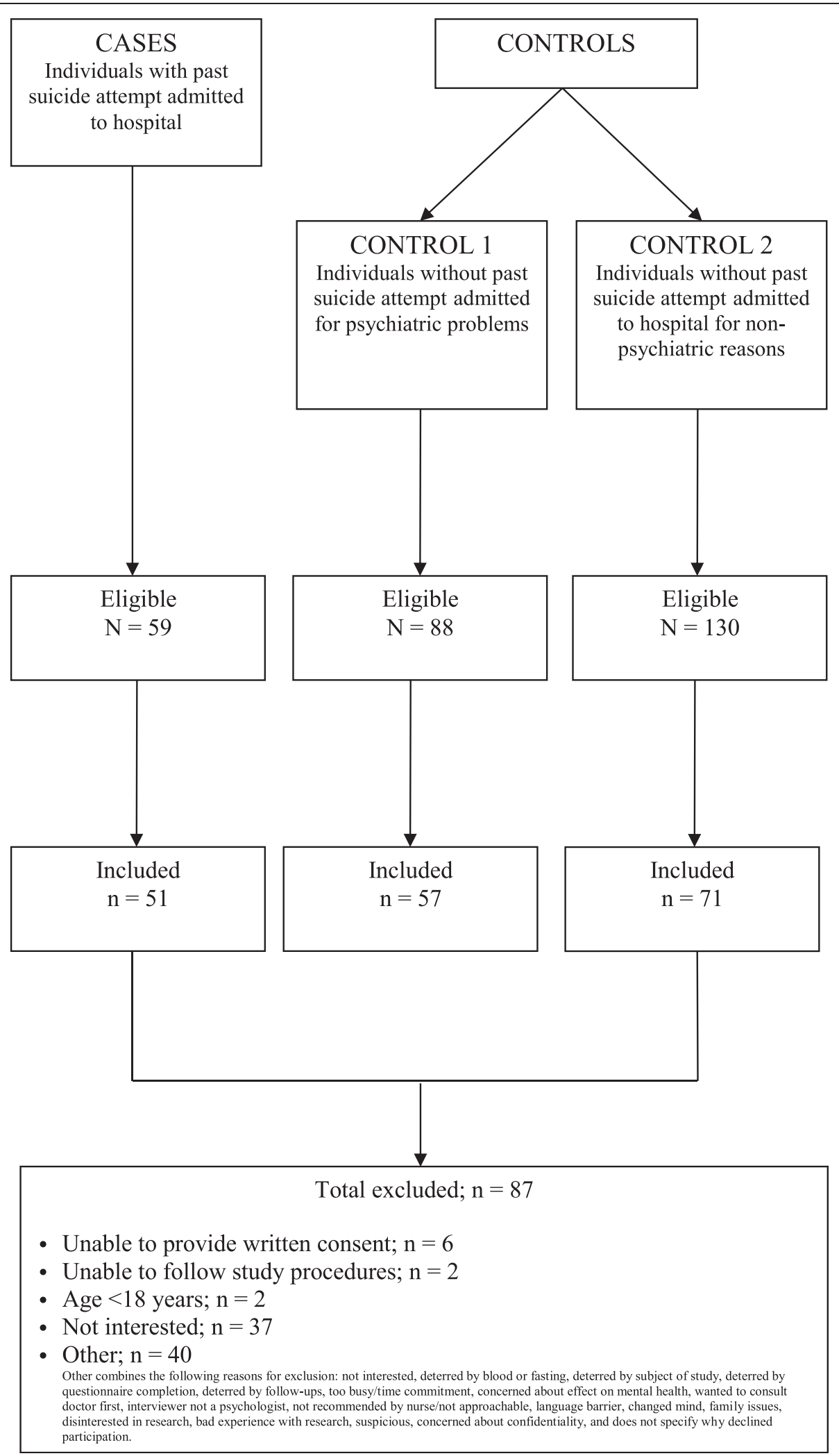

Fig. 2 Flow diagram for participants included in study 
Table 1 Characteristics of study participants $(n=179)$

\begin{tabular}{|c|c|c|c|c|}
\hline Variable & Case $(n=51)$ & Control $1(n=57)$ & Control $2(n=71)$ & $\begin{array}{l}P \text { (ANOVA for mean difference, } \\
X^{2} \text { for categorical variables) }\end{array}$ \\
\hline Age in years; mean (SD) & $43.2(13.3)$ & $45.3(14.0)$ & $46.7(18.7)$ & 0.529 \\
\hline Female; $n(\%)$ & $24(47.1)$ & $29(50.9)$ & $34(47.9)$ & 0.913 \\
\hline $\operatorname{BMI}\left(\mathrm{kg} / \mathrm{m}^{2}\right) ;$ mean (SD) & $28.3(8.5)$ & $28.7(5.7)$ & $28.3(6.5)$ & 0.097 \\
\hline Never married; $n$ (\%) & $19(37.3)$ & $23(40.4)$ & $22(31.0)$ & 0.028 \\
\hline Completed college/university; $n$ (\%) & $20(37.3)$ & $21(36.8)$ & $41(57.7)$ & 0.017 \\
\hline Currently employed; $n$ (\%) & $12(23.5)$ & $19(33.9)$ & $39(54.9)$ & 0.001 \\
\hline Annual income <30 k/year; $n(\%)$ & $20(39.2)$ & $21(36.8)$ & $12(16.9)$ & 0.051 \\
\hline Currently smoking; $n(\%)$ & $25(49.0)$ & 18 (31.6) & $10(14.1)$ & $<0.001$ \\
\hline Family history of suicidal behavior; $n(\%)$ & $20(39.2)$ & $16(28.1)$ & $17(23.9)$ & 0.081 \\
\hline
\end{tabular}

BMI body mass index, ANOVA analysis of variance

analyses presented in this study are strictly exploratory. The reporting of $p$ values and any other significant results should be interpreted with caution.

\section{Key learning points from the pilot experience}

Our study aims to investigate several risk factors for suicidal behavior; therefore, the number of assessments required to collect the information we need is extensive. We found that administering the full questionnaire was time-consuming for the interviewer and in some instances participants were unable to stay focused for the duration of the interview or had other scheduled tasks to attend to. Therefore, we modified the study procedures and conducted the interview over two sessions or as requested at the convenience of the participants.

During the course of the pilot recruitment, we found that many participants have been in the hospital under close supervision for a long duration prior to the study enrolment and therefore were not eligible to participate in the study due to timing of the 1-month inclusion criterion. This led us to revise our inclusion criterion of recent suicide attempt to include the 3 months prior to the study enrolment; however, potential cases would have remained in hospital at the time of study recruitment.

We learned that participants were having difficulty answering questions from the Borderline Symptom List because of the response options provided (i.e., not at all, a little, rather, much, very strong). Therefore, we provided alternative wording and explanations of the Likert scale. The Social Support Questionnaire seemed to be repetitive as participants generally reported one consistent source of support, and therefore, we will ask about the main source of support and avoid repeating the questions to participants.

\section{Study strengths and limitations}

The study design we have chosen to investigate suicidal behavior risk factors is a case-control design where risk factors are compared among cases and controls. Ideally a cohort study design will be chosen to investigate risk factors; however, for the suicide risk factors study, the case control design is the most suitable based on the relatively low incidence of the suicidal behavior in the population (110/100,000 for suicide attempts). We would also need very large sample size and prolonged follow-up duration to acquire adequate number of cases, making a cohort design prohibitive to achieve. We believe that the case-control study design is the most efficient way to provide reliable information on the importance of several suicide risk factors when potential biases and confounders are minimized. By implementing a case-control study design, we have kept costs at a minimum and also reduced the possibility of losing participants to follow-up, which would be problematic with other study designs. We have

Table 2 Criteria for success of feasibility and feasibility outcomes

\begin{tabular}{lll}
\hline Feasibility outcome & Criterion & Results \\
\hline Participant consent & $50 \%$ of all eligible cases consent to participate & $\begin{array}{l}70 \% \text { recruitment rate (213 participants out of } \\
304 \text { approached) } \\
\text { Number of participants recruited }\end{array}$ \\
$\begin{array}{l}50 \text { cases and } 100 \text { controls per year can be recruited } \\
\text { Collection of blood samples }\end{array}$ & $80 \%$ of the participants will provide blood samples & $\begin{array}{l}90 \% \text { provided blood samples (191 out of } 213 \\
\text { participants) }\end{array}$ \\
\hline
\end{tabular}




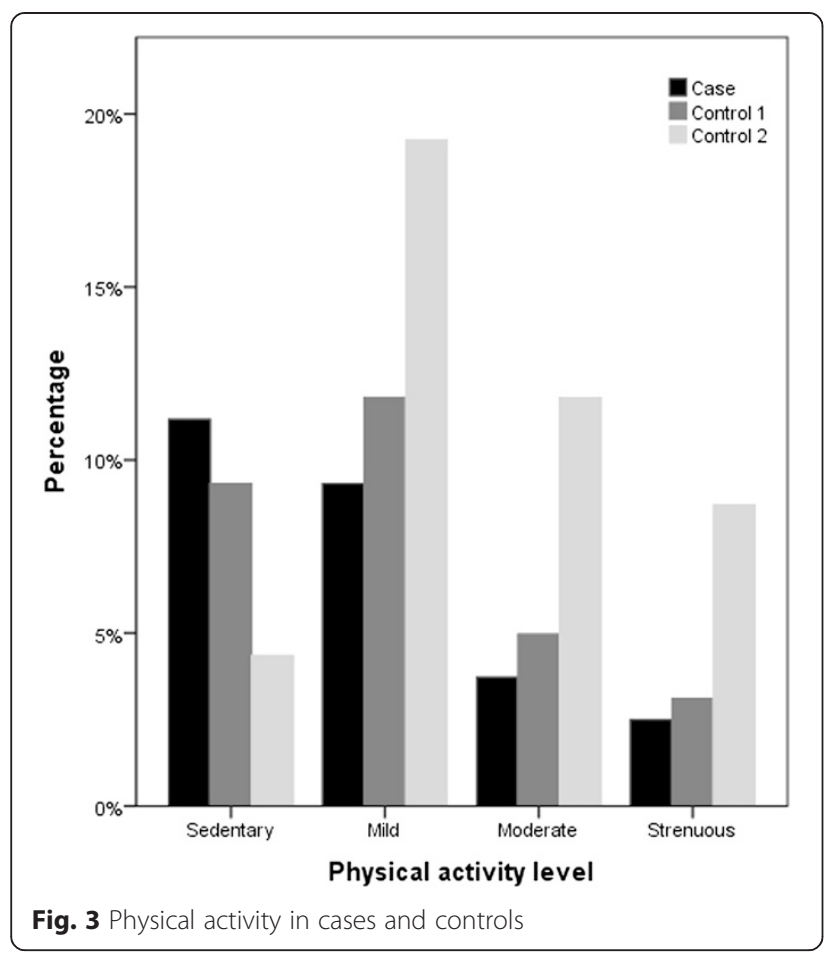

also chosen to include two control groups so that more reliable comparisons can be drawn. However, the casecontrol study design is not without limitations. The results of any case-control study may be influenced by bias. In the case of social desirability, cases may under- report adverse lifestyle behaviors compared to controls or over-report advantageous lifestyle behaviors.

\section{Future directions}

Recruitment for the full-scale DISCOVER study is underway, and we have incorporated several changes based on the results of this pilot study. First, we have modified the questionnaire according to our findings about the Borderline Symptom List, the Social Support Questionnaire, and the overall length of the interview. We also decided to stop collecting blood pressure and pulse rate as these measures are not risk factors or confounding factors for suicide attempt. After our preliminary results showed interesting results for the food patterns between cases and controls, we administered a comprehensive Food Frequency Questionnaire to measure participant habitual intake.

For the full-scale study, our ultimate goal is to conduct a prospective case-control study. We aim to include 100 individuals with recent suicide attempts requiring hospitalization and 200 controls with a yearly follow up of 5 years to record any new suicidal behavior events. The sample size for the study was conservatively estimated based on the recruitment rate and the relatively rare event of suicidal behavior requiring hospitalization.

\section{Conclusions}

This pilot study examined the feasibility of the DISCOVER full-scale study, and we are able to conclude that it is feasible to conduct the full study with some modifications.

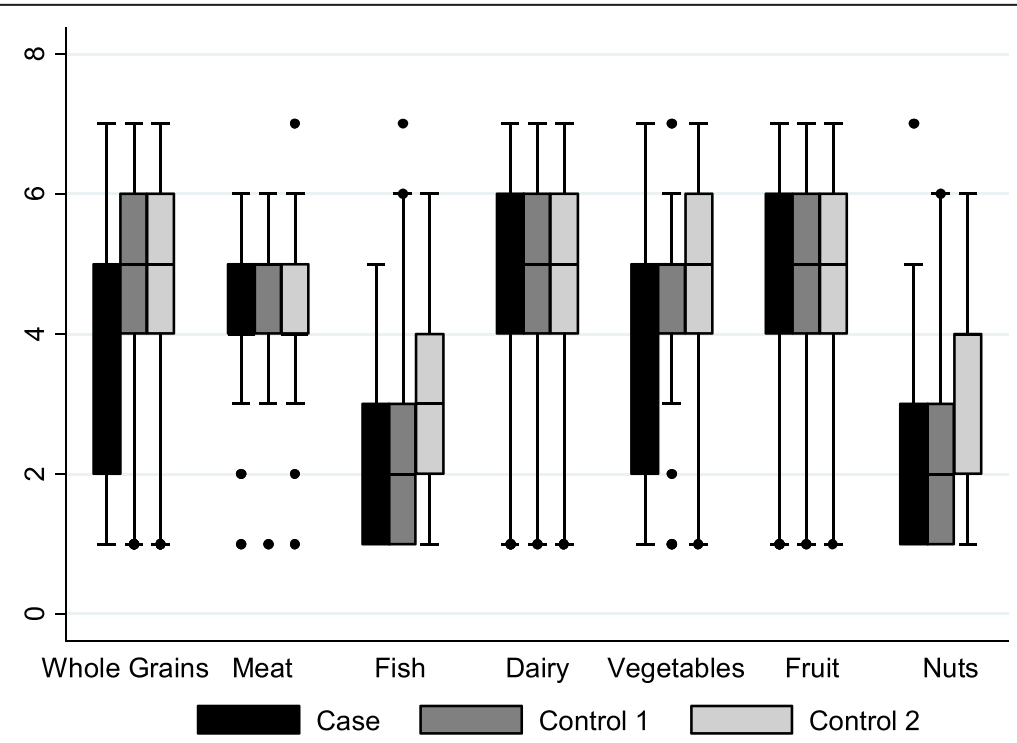

Fig. 4 Mean number of servings of food groups by cases and controls. Legend for Fig. 4: The error lines represent variance around the mean estimates for number of food servings. These lines extend to the outer fences of interquartile range, which is represented by the size of the box. Any dots in this image represent outlier observations 


\section{Additional file}

Additional file 1: Table S1. Comparative Demographic Characteristics Between Cases and Control Group 1. Table S2 Comparative

Demographic Characteristics Between Cases and Control Group 2.

\section{Competing interests}

The authors declare that they have no competing interests.

\section{Authors' contributions}

All authors contributed to the development of this paper. ZS conceived the study and she is principally responsible for the conduct of the study, obtaining funding and writing of manuscripts. $\mathrm{MB}$ and $\mathrm{BD}$ assisted with recruitment and wrote the first draft of this manuscript under ZS supervision. WE-S is the research assistant for this study and is responsible for recruitment, interviews, data collection, and weekly team meeting preparations. JD is the study coordinator responsible for the daily running of the study, training research assistants and study personnel on study-related procedures. SR is the study project manager and is responsible for the study conduct; with the PI (ZS) she designed the study protocol, provided supervision of study personnel, and coordinated the overall study management. JV, HS, NH, and $\mathrm{El}$ are nurses specializing in psychiatry and practicing at psychiatric hospital; they conducted interviews, collected blood samples, recruited hospitalized study participants, and provided feedback on participants' recruitment and feedback on the study procedures. PM is responsible for database management, quality and edits, checks of data and provision of reports, including age and sex matching status, to the study team for the weekly study meetings. SI is responsible for data analysis and preparation of data reports to the team. MD is responsible for the inclusion and analysis of the diet questionnaire. JB provided advice on study procedures including recruitment and the inclusion of psychiatric controls based on her expertise in emergency psychiatry and suicide prevention. LT is responsible for the case-control study design, and overall methodological and statistical aspects of the study. LT is also responsible for the design and reporting of the pilot and feasibility study. All authors have reviewed and approved the final version of the manuscript.

\section{Acknowledgements}

The authors wish to thank the study participants; without their generous contributions, it would not have been possible to conduct this study. Our sincere thanks also go to Hamilton Health Sciences and St. Joseph's Healthcare Hamilton hospitals and managers who facilitated the study and provided valuable feedback throughout the study period. We would like to thank many McMaster undergraduate students who have helped and continue to help in the study including Bianca Bantoto, Shireen Aslam, Alison Zarouk and Marta Sadwoski, and PHRI staff including Leah Heldman, and Amparo Casanova.

This work was supported by PHRI and The Brain and Behaviour Research Foundation Young Investigator Grant (\# 19058).

\section{Author details}

${ }^{1}$ Department of Psychiatry and Behavioural Neurosciences, McMaster University, Hamilton, ON, Canada. ²Department of Clinical Epidemiology and Biostatistics, McMaster University, Hamilton, ON, Canada. ${ }^{3}$ Population Genomics Program, Chanchlani Research Centre, McMaster University, Hamilton, ON, Canada. ${ }^{4}$ MiNDS Neuroscience Program, McMaster University, Hamilton, ON, Canada. ${ }^{5}$ Population Health Research Institute, Hamilton Health Sciences/McMaster University, Hamilton, ON, Canada. ${ }^{6}$ St. Joseph's Healthcare Hamilton, 100 West 5th Street, Hamilton, ON L8N 3K7, Canada. ${ }^{7}$ Biostatistics Unit, Centre for Evaluation of Medicine, Hamilton, ON, Canada. ${ }^{8}$ System Linked Research Unit, Hamilton, ON, Canada.

Received: 20 October 2014 Accepted: 1 May 2015 Published online: 09 May 2015

\section{References}

1. Krug E, Dahlberg L, Mercy J, Zwi A, Lozano R. World report on violence and health. Geneva: World Health Organization; 2002

2. Värnik P. Suicide in the world. Int J Environ Res Public Health. 2012;9(3):760-71.

3. Mann JJ. Neurobiology of suicidal behaviour. Nat Rev Neurosci. 2003;4(10):819-28
4. De Moore GM, Robertson AR. Suicide in the 18 years after deliberate self-harm a prospective study. Br J Psychiatry. 1996;169(4):489-94.

5. Jenkins GR, Hale R, Papanastassiou M, Crawford MJ, Tyrer P. Suicide rate 22 years after parasuicide: cohort study, vol. 325. 2002

6. Suominen K, Isometsa E, Suokas J, Haukka J, Achte K, Lonnqvist J. Completed suicide after a suicide attempt: a 37-year follow-up study. Am J Psychiatry. 2004;161(3):562-3.

7. Beautrais A, Joyce P, Mulder R, Fergusson D, Deavoll B, Nightingale S. Prevalence and comorbidity of mental disorders in persons making serious suicide attempts: a case-control study. Am J Psychiatry. 1996;153(8):1009-14.

8. Muldoon MF, Manuck SB, Matthews KA. Lowering cholesterol concentrations and mortality: a quantitative review of primary prevention trials. BMJ. 1990;301(6747):309-14.

9. Alvarez JC, Cremniter D, Gluck N, Quintin P, Leboyer M, Berlin I, et al. Low serum cholesterol in violent but not in non-violent suicide attempters. Psychiatry Res. 2000;95:103-8.

10. Ellison LF, Morrison HI. Low serum cholesterol concentration and risk of suicide. Epidemiology. 2001;12:168-72.

11. Kim Y-K, Lee H-J, Kim J-Y, Yoon D-K, Choi S-H, Lee M-S. Low serum cholesterol is correlated to suicidality in a Korean sample. Acta Psychiatr Scand. 2002;105(2):141-8.

12. Lee H-J, Kim Y-K. Serum lipid levels and suicide attempts. Acta Psychiatr Scand. 2003;108(3):215-21.

13. Loncar C, Definis-Gojanovic M, Dodig G, Jakovljevic M, Franic T, Marcinko D, et al. War, mental disorder and suicide. Coll Antropol. 2004;28(1):377-84.

14. Huan M, Hamazaki K, Sun Y, Itomura M, Liu H, Kang W, et al. Suicide attempt and $\mathrm{n}-3$ fatty acid levels in red blood cells: a case control study in china. Biol Psychiatry. 2004;56(7):490-6.

15. Tanskanen A, Hibbeln JR, Hintikka J, Haatainen K, Honkalampi K, Viinamaki H, et al. Fish consumption, depression, and suicidality in a general population. Arch Gen Psychiatry. 2001;58(5):512-3.

16. Davidson CL, Babson KA, Bonn-Miller MO, Souter T, Vannoy S. The impact of exercise on suicide risk: examining pathways through depression, PTSD, and sleep in an inpatient sample of veterans. Suicide Life Threat Behav. 2013;43(3):279-89.

17. O'Neill S, Corry CV, Murphy S, Bunting BP. Characteristics of deaths by suicide in northern Ireland from 2005-2011 and use of health services prior to death. J Affect Disord 2014.

18. Clayden R, Zaruk A, Meyre D, Thabane L, Samaan Z. The association of attempted suicide with genetic variants in the SLC6A4 and TPH genes depends on the definition of suicidal behavior: a systematic review and meta-analysis. Transl Psychiatry. 2012;2(10):e166.

19. Kessler RC, Borges $\mathrm{G}$, Walters EE. Prevalence of and risk factors for lifetime suicide attempts in the National Comorbidity Survey. Arch Gen Psychiatry. 1999;56(7):617-26.

20. Qin P, Agerbo E, Mortensen PB. Suicide risk in relation to socioeconomic, demographic, psychiatric, and familial factors: a national register-based study of all suicides in Denmark, 1981-1997. Am J Psychiatry. 2003; 160(4):765-72.

21. Brown GK, Beck AT, Steer RA, Grisham JR. Risk factors for suicide in psychiatric outpatients: a 20-year prospective study. J Consult Clin Psychol. 2000;68(3):371-7.

22. Bach H, Huang Y-Y, Underwood MD, Dwork AJ, Mann JJ, Arango V. Elevated serotonin and 5-HIAA in the brainstem and lower serotonin turnover in the prefrontal cortex of suicides. Synapse. 2014;68(3):127-30.

23. Park Y-M, Lee B-H, Um TH, Kim S. Serum BDNF levels in relation to illness severity, suicide attempts, and central serotonin activity in patients with major depressive disorder: a pilot study. PLoS One. 2014;9(3):e91061.

24. Janelidze S, Ventorp F, Erhardt S, Hansson O, Minthon L, Flax J, et al. Altered chemokine levels in the cerebrospinal fluid and plasma of suicide attempters. Psychoneuroendocrinology. 2013;38(6):853-62.

25. Lucas M, O'Reilly EJ, Pan A, Mirzaei F, Willett WC, Okereke Ol, et al. Coffee, caffeine, and risk of completed suicide: results from three prospective cohorts of American adults. World J Biol Psychiatry. 2013;(0):1-10.

26. Alvarado-Esquivel C, Hern ${ }^{\circ}$ ndez-Tinoco J, S ${ }^{\circ}$ nchez-Anguiano LF, Arnaud-Gil CA, Molina-Espinoza LF. Eating habits associated with a history of suicide attempts in psychiatric outpatients. Health. 2014;6:317.

27. Umhau JC, George DT, Heaney RP, Lewis MD, Ursano RJ, Heilig M, et al. Low vitamin D status and suicide: a case-control study of active duty military service members. PLoS One. 2013;8(1):e51543. 
28. Lucas MO, Ä̂̂Reilly EJ, Mirzaei F, Okereke Ol, Unger L, Miller M, et al. Cigarette smoking and completed suicide: results from 3 prospective cohorts of American adults. J Affect Disord. 2013;151(3):1053-8.

29. Taliaferro LA, Rienzo BA, Pigg RM, Miller MD, Dodd VJ. Associations between physical activity and reduced rates of hopelessness, depression, and suicidal behavior among college students. J Am Coll Health. 2009;57(4):427-36.

30. Thabane L, Ma J, Chu R, Cheng J, Ismaila A, Rios L, et al. A tutorial on pilot studies: the what, why and how. BMC Med Res Methodol. 2010;10(1):1.

31. Beck AT, Schuyler D, Herman I. Development of suicidal intent scales. In: Beck AT, Resnik HLP, Lettieri DJ, editors. The prediction of suicide, vol. 47. Bowie, MD: Charles Press; 1974.

32. Sheehan DV, Lecrubier $Y$, Sheehan $\mathrm{KH}$, Amorim $P$, Janavs J, Weiller $E$, et al. The Mini-International Neuropsychiatric Interview (M.I.N.I.): the development and validation of a structured diagnostic psychiatric interview for DSM-IV and ICD-10. J Clin Psychiatry. 1998;59 Suppl 20:22-33.

33. Patton JH, Stanford MS, Barratt ES. Factor structure of the Barratt impulsiveness scale. J Clin Psychol. 1995;51:768-74.

34. Sarason IG, Levine HM, Basham RB, Sarason BR. Assessing social support: the social support questionnaire. J Pers Soc Psychol. 1983;44:127-39.

35. Bohus M, Kleindienst N, Limberger MF, Stieglitz RD, Domsalla M, Chapman $A L$, et al. The short version of the Borderline Symptom List (BSL-23): development and initial data on psychometric properties. Psychopathology. 2009;42(1):32-9.

36. Hallal PC, Victora CG. Reliability and validity of the International Physical Activity Questionnaire (IPAQ). Med Sci Sports Exerc. 2004;36(3):556.

37. Hagstromer M, Oja P, Sjostrom M. The International Physical Activity Questionnaire (IPAQ): a study of concurrent and construct validity. Public Health Nutr. 2006;9(6):755-62

38. Iqbal R, Anand S, Ounpuu S, Islam S, Zhang X, Rangarajan S, et al. Dietary patterns and the risk of acute myocardial infarction in 52 countries: results of the INTERHEART study. Circulation. 2008;118(19):1929-37.

\section{Submit your next manuscript to BioMed Central and take full advantage of:}

- Convenient online submission

- Thorough peer review

- No space constraints or color figure charges

- Immediate publication on acceptance

- Inclusion in PubMed, CAS, Scopus and Google Scholar

- Research which is freely available for redistribution 